\section{Ya se dispone de antirretrovíricos contra el VIH y el sida: ¿Es necesaria ahora la prevención?}

\author{
Peter Aggleton ${ }^{1}$ y Rafael Mazín ${ }^{2}$
}

Hasta hace poco tiempo, la única estrategia disponible contra el virus de la inmunodeficiencia humana (VIH) se basaba en actividades de prevención. De hecho, el hoy inexistente Programa Mundial del Sida (PMS) de la Organización Mundial de la Salud ponía de relieve la necesidad inaplazable de contener el avance de la epidemia mediante acciones dirigidas a prevenir la infección. Como ejemplo podría señalarse que en sus publicaciones, a manera de cliché, el PMS declaraba que "en ausencia de un tratamiento o vacuna efectivos, la única solución [que quedaba era] la prevención". De este modo, paradójica e inadvertidamente se adjudicaba a la prevención un valor muy relativo, dado más que nada por la ausencia de una mejor alternativa. Curiosamente, tal manera de enfocar la prevención no causó mayor sorpresa en diversos niveles de la sociedad, porque todo el mundo realmente esperaba soluciones reparadoras para este problema emergente. Sin duda la prevención se consideraba más o menos un remedio paliativo o un placebo, algo "mejor que nada", pero de ninguna manera "la solución".

\section{La prevención sigue rezagada}

Pese a que en los últimos años se ha venido subrayando la importancia del enfoque preventivo mediante acciones de promoción y protección de la salud, la verdad es que en materia de atención de la salud en todos los niveles de la sociedad continúa prevaleciendo el "paradigma curativo-reparador" de la medicina. Ejemplo de ello es que la inmensa mayoría de las personas siguen acudiendo a los servicios de salud no a recibir orientación sobre formas de preservar la salud, sino buscando que le alivien una dolencia o le resuelvan algún problema físico. En muchos casos dichos trastornos de salud pudieron haberse evitado y no es raro que la gente llegue demasiado tarde en busca del remedio ansiado.

La situación tiende a perpetuarse porque la estructura de los sistemas de salud está hecha "a la medida" del mencionado paradigma "curativoreparador". En hospitales y clínicas se aplica la "ciencia dura", en tanto que el trabajo sanitario en otros contextos (por ejemplo, a nivel comunitario) se concibe como de una índole diferente. Inclusive, en la mayor parte de las escuelas de medicina la formación del futuro profesional sigue enfocándose en el 
aprendizaje de cuadros nosológicos y patológicos, así como en las estrategias farmacológicas y quirúrgicas recomendables para curarlos o repararlos.

Para el estudiante de medicina, la prevención no resulta tan fascinante como la dinámica de la sala de emergencias o los servicios clínicos. Tal vez por ello el futuro médico encuentre reconfortante que en los programas de estudio de la carrera los contenidos de medicina preventiva y salud pública solo se aborden formalmente, si acaso, durante unas cuantas semanas. El interés de la mayoría de los estudiantes parecería inclinarse más hacia el aprendizaje de la semiología, la patología y la farmacología, muy probablemente porque encuentran más interesante el desafío de realizar diagnósticos y recomendar tratamientos. Más aún, el perfil heroico del profesional de la salud que tanto contribuyó a consolidar la literatura romántica primero y más tarde los seriados de televisión sobre abnegados, atractivos y exitosos médicos, es más patente y más dramático cuando tiene que lidiar con el dolor, la enfermedad y la muerte que cuando se esfuerza por "alejar al enemigo, en vez de combatirlo cara a cara", como propugna el enfoque preventivo.

La prevención basada en la promoción de la salud para el desarrollo de comportamientos de menor riesgo se realza en los discursos, en los documentos de políticas y en resoluciones, pero en la práctica se sigue considerando como un recurso de segunda — cuando no de tercera- categoría, una última opción para cuando no hay vacunas ni medicamentos disponibles. No debe extrañar entonces que, desde los albores de la respuesta organizada contra el sida, se exigiera el empeño de todos los recursos financieros y científicos necesarios para la búsqueda de soluciones tangibles y "veraderamente eficaces", es decir, de medicamentos para curar la enfermedad.

Los productos farmacéuticos son, en opinión del público $-\mathrm{y}$ de no pocos profesionales de la salud-las únicas opciones eficaces, porque permiten resolver el problema de una vez por todas. Para el común de la gente las respuestas que ofrezca la ciencia ante los problemas de salud deben ser ingeribles o inyectables. Cualquier alternativa se considera una mera formalidad sin mayor importancia, un marco necesario para encuadrar el "único y verdadero remedio": el fármaco. Las sugerencias del profesional de la salud que se relacionan con hábitos o comportamientos del paciente (e. g., "trate de relajarse", "coma bien", "deje de fumar", etc.) suelen considerarse exhortaciones distantes, obvias y vagas. Lo importante es la prescripción de cualquier producto farmacéutico porque la gente piensa que "eso es lo único que realmente cura".

No es, pues, de extrañar que las expectativas del público en general estén básicamente centradas en descubrimientos que pudieran resolver el problema mediante elementos externos de valor casi mágico. Sin duda alguna la disponibilidad de un medicamento se percibe como una respuesta mucho más conveniente y eficaz que aquella que exige esfuerzos individuales de prevención usualmente fastidiosos y complicados. Por lo mismo no sorprende que cuando un periodista, un obrero, un abogado o cualquiera de las personas que integran el denominado "público general" descubre que su interlocutor es un experto en sida, surjan preguntas como ¿cuándo habrá una vacuna o tratamiento? o ¿cuánto se ha avanzado para conseguir el remedio?, o bien, "¿cómo es posible que con todos los avances aún no se haya descubierto una medicina eficaz?".

Este último comentario refleja la idea, bastante generalizada, de que el desarrollo de la ciencia y la tecnología ha llegado a tal nivel de complejidad que no es posible que no haya respuestas para prácticamente cualquier desafío. Tal convencimiento permite entender cómo ciertas actitudes de aparente indiferencia ante la posibilidad de infectarse, más que cimentarse en apatía o en desidia, parezcan más bien sustentarse en una confianza ciega en lo mucho que ha avanzado la ciencia y la rapidez con que lo sigue haciendo. Partiendo de dicha premisa, para el observador externo la aparente falta de preocupación con el riesgo implícito en ciertas prácticas no resulta tan irracional como pudiera parecer a primera vista. Por cierto, hay una racionalidad subyacente en la confianza absoluta que la gente deposita en la ciencia y la tecnología, porque los avances están ocurriendo y los medios de comunicación dan cuenta de ellos.

En cuanto a la infección por el VIH, hasta muy recientemente los mayores progresos se habían obtenido casi de forma exclusiva en el manejo clínico de las infecciones oportunistas. Los fármacos antirretrovirales disponibles no parecían tener mayor efecto que el de desacelerar levemente la progresión de la enfermedad por el VIH y, desde luego, faltaba identificar una vacuna realmente eficaz. Sin embargo, en 1996 (durante la XI Conferencia Internacional sobre Sida, celebrada en Vancouver, Canadá) se anunciaron los resultados de un esquema combinado de tratamiento con tres fármacos antirretrovíricos. El aspecto más halagüeño de este esquema terapéutico era que su utilización sistemática durante un período determinado reducía las concentraciones plasmáticas de VIH a niveles muy bajos o prácticamente no detectables, con una notable mejoría de las condiciones del paciente. Esto permitía vislumbrar la posibilidad de un tratamiento eficaz y quizás la cura para la enfermedad provocada por el virus.

La noticia se difundió rápidamente y produjo una demanda generalizada, exigiéndose que los 
nuevos tratamientos se hicieran accesibles para todos. De igual manera se exigía una mayor inversión de recursos financieros que permitieran redoblar los esfuerzos en materia de investigación para conseguir la máxima eficacia de los medicamentos antirretrovíricos. El empleo generalizado en países industrializados de los esquemas combinados de tratamiento con fármacos antirretrovíricos, en particular aquellos que incorporaron los recién descubiertos inhibidores de la proteasa vírica, confirmó pronto que estos eran realmente muy eficaces en la reducción de la cantidad de partículas víricas a niveles casi indetectables en muchos pacientes. Como resultado, se comenzó a observar un mejoramiento en los cuadros clínicos y en la calidad y la duración de la vida de las personas infectadas por VIH. Algunos autores señalaron que existía la posibilidad teórica de erradicar totalmente hasta el último vestigio del virus en el organismo de las personas infectadas y de lograr una curación completa.

Visto retrospectivamente, el optimismo resultó prematuro. En la XII Conferencia Internacional sobre Sida (llevada a cabo a comienzos de julio de 1998 en Ginebra, Suiza) se realizó una evaluación en un tono más cauteloso que el usado en las declaraciones efectuadas dos años atrás. Se difundieron noticias sobre nuevos avances en los tratamientos, pero también se dieron a conocer informes sobre el desarrollo de resistencia a dichos tratamientos, efectos colaterales imprevistos, dificultades para aplicar esquemas de procedimiento con medicamentos múltiples y costos prohibitivos. El entusiasmo desbordante generado en Vancouver se transformó en un súbito desencanto con los resultados anunciados en Ginebra.

A un año de la Conferencia de Ginebra se habían acumulado bastantes pruebas de que no todos aquellos que reciben terapia combinada obtienen los beneficios esperados y que aun entre aquellos que experimentan una notable mejoría, algunos virus sobreviven la terapia "ocultándose" en ciertos órganos y tejidos donde los fármacos antirretrovíricos no penetran, por lo que no puede considerase que estos esquemas terapéuticos tengan un valor curativo definitivo $(19,20)$. Las recomendaciones y prácticas acerca del uso de los fármacos antirretrovíricos cambian, de hecho, muy rápidamente y es de esperar que continúen haciéndolo en el futuro próximo.

Es muy factible que el principal motivo de desencanto con los esquemas de terapia antirretrovírica combinada radique en el reconocimiento de que en la mayor parte de los países el tratamiento no está disponible ni lo estará en el futuro cercano, entre otras causas por su elevado costo. Como si no fuera suficiente, su uso requiere una infraestructura tecnológica que no siempre se encuentra disponible en países en desarrollo, donde lamentablemente se concentran $90 \%$ de los casos de sida.

Aun cuando casi todos los días se anuncia un hallazgo importante en la investigación sobre el $\mathrm{VIH} /$ sida y las iniciativas para desarrollar vacunas efectivas siguen su curso, cabe preguntarse si tales avances realmente servirán a "toda la humanidad" o serán solo el privilegio de quienes puedan pagarlos. Mientras tanto, no cabe duda que las mejores oportunidades para el control de la pandemia las ofrece la prevención basada en la reducción de riesgos, mediante acciones de promoción y protección de la salud. En otras palabras, la prevención sigue y seguirá siendo la primera línea para el control de la epidemia de VIH y, de ninguna manera, el último recurso para enfrentarla.

\section{¿Cómo lograr que las iniciativas de prevención resulten más efectivas?}

La experiencia internacional acumulada en los últimos años ha demostrado que el éxito de los programas de prevención y control de la infección por VIH (1) depende en gran medida de los siguientes componentes: voluntad política para hacer frente a la epidemia; diseminación masiva de información sobre VIH/sida; actividades orientadas a grupos y comunidades particularmente vulnerables; amplio acceso a condones y otros materiales de higiene, como agujas y jeringas esterilizadas; y provisión de servicios de calidad para el tratamiento de las enfermedades de transmisión sexual (2).

La exclusión de cualquiera de estos elementos - o el no tomar en cuenta metódicamente las características y necesidades locales específicas- debilita de manera drástica el impacto de los programas. En cambio, cuando se conjugan todos y cada uno de estos componentes, se ha conseguido un esfuerzo de reducción de riesgos que ha demostrado ser efectivo para controlar la epidemia en diferentes países del mundo.

La voluntad política constituye un requisito para el establecimiento de un ambiente propicio donde habrán de desarrollarse acciones dirigidas a la prevención. Las decisiones en la esfera política, sin embargo, no son tomadas desde un punto de vista estrictamente técnico, sino como resultado de una mezcla de concesiones ideológicas, argumentos técnicos, impacto económico y, sobre todo, la posibilidad de demostrar los resultados logrados con las decisiones tomadas. En términos generales, las pruebas en torno a los resultados de la implementación de acciones preventivas son meras especulaciones que describen escenarios hipotéticos pero que fueron modificados en sentido positivo; 
situaciones evitadas; peligros desviados; riesgos alejados. Resulta más convincente, por lo tanto, mostrar el compromiso político en forma de determinar la cantidad de productos farmacológicos distribuidos para tratar un número específico de casos, que mostrar acciones preventivas emprendidas en cierto período, que (tal vez) previnieron la aparición de determinado número de casos nuevos.

Por otro lado, emprender acciones preventivas exige abordar temas que habitualmente se consideran "espinosos" (e.g., relaciones premaritales y extramaritales, homosexualidad, uso de drogas, etc.). Para los políticos deseosos de evitar conflictos con los sectores más conservadores de la sociedad (a menudo con una fuerte influencia económica) es preferible buscar refugio en el terreno menos polémico del cuidado de las personas que ya se encuentran enfermas. Distribuir medicamentos será una acción bien vista por muchas personas, mientras que apoyar acciones para distribuir condones entre adolescentes $\mathrm{u}$ homosexuales, o agujas y jeringas entre usuarios de drogas, puede acarrear críticas y malestar. La evasión flagrante del conflicto con las alas conservadoras de la sociedad se facilita enormemente en un medio dominado por un paradigma "curativo y reparador" de la medicina. Es necesario un esfuerzo especial de parte de los expertos en salud pública y educación en salud para persuadir a los responsables de las decisiones políticas de los beneficios y ventajas de la inversión en medidas preventivas.

Para ello resulta de fundamental importancia desarrollar acciones educativas que sirvan para hacer frente a un pretendido "sentido común" que sustenta ciertas ideas y creencias. Ejemplos de esa supuesta "sabiduría secular" son las creencias de que la educación sexual incita a los jóvenes a la exploración del sexo; que la homosexualidad puede ser promovida; que la distribución de agujas estériles aumenta el consumo de drogas; que los jóvenes estarán libres de riesgo si se les habla solo de la abstinencia, y que la medicina tiene al alcance una solución definitiva para cualquier problema. Igualmente importante es colaborar directamente con los responsables de las políticas en el diseño de instrumentos y métodos para conseguir pruebas tangibles y cuantificables del desarrollo de intervenciones. Varios modelos, ya en uso en la actualidad, pueden servir para demostrar al contribuyente que el dinero de sus impuestos está tan bien empleado cuando se invierte en acciones de prevención, como cuando se gasta en construir hospitales o distribuir medicamentos. Además, dotar a los responsables de las decisiones políticas de datos sólidos ayuda a disipar los temores creados por la ignorancia, el oscurantismo y la intolerancia.

\section{¿Cómo afecta al comportamiento la terapia antirretrovírica muy activa?}

El descubrimiento de la terapia antirretrovírica muy activa (ARMA, conocida en inglés como HAART) ha generado nuevos y grandes retos para la prevención de la infección por VIH, entre ellos el relacionado con la percepción errada de que dicha infección y el sida son tratables y curables. Esta nueva percepción parece afectar a la determinación de las personas de mantener relaciones sexuales de bajo riesgo o relaciones sexuales seguras.

Si bien se sabe que la terapia no es accesible ni apropiada para todos ni es curativa para nadie, hay pruebas de que tanto en los Estados Unidos como en Australia y Francia el optimismo producido por los tratamientos está empezando a alterar la percepción de la gravedad del VIH/sida. A principios de 1997, James Dilley y varios colegas observaron que alrededor de la cuarta parte de los hombres homosexuales entrevistados en San Francisco decían estar "menos preocupados de volverse seropositivos debido a los nuevos tratamientos". A la pregunta "¿Está más dispuesto a correr el riesgo de quedar infectado al tener relaciones sexuales?", $13 \%$ respondieron "de alguna manera" o "fuertemente" (4).

Según encuestas cruzadas realizadas en Australia entre hombres homosexuales de Sidney, mientras que en febrero de $199614,9 \%$ de los encuestados declaraban que durante los últimos 6 meses habían realizado coito anal sin protección con parejas casuales, en el mismo lapso previo a agosto de 1997 el porcentaje había ascendido a 26,1\% (3). Datos recientes del Male Call 96 —una encuesta telefónica nacional realizada en Australia entre hombres que han tenido relaciones sexuales con otros hombres- mostraron una tendencia similar, aunque menos pronunciada. En la muestra -mucho más numerosa- las tasas totales de coito anal sin protección aumentaron de $12 \%$ en 1992 a 15\% en 1996.

Existe gran variedad de razones que pueden explicar este incremento en el coito anal desprotegido, entre ellas el optimismo por el éxito obtenido en los tratamientos; el aumento de las relaciones sexuales en parejas cuyos miembros son ambos seropositivos; la interpretación por parte de algunos hombres de la expresión "carga vírica no detectable" como ausencia de infección; la reducción de la efectividad de los mensajes sobre el sexo seguro, y la posibilidad de que ciertas campañas recientes sobre la actividad sexual sin riesgo hayan facilitado a algunos hombres homosexuales describir cándidamente su conducta (5). Seguramente la confianza absoluta de que la ciencia "ahora sí se encuentra muy cerca de la cura" puede ser un incentivo para 
no privarse de la relación sexual desprotegida. "Al final de cuentas, si llego a infectarme, cuando comience a tener síntomas ya tendrá que haber un remedio disponible" podría ser la racionalización para un comportamiento que proporciona satisfacción emocional y física, aunque encierre grandes riesgos.

Independientemente de cuál de esas razones sea la más plausible, vale la pena destacar que en Australia, mientras el coito anal ha aumentado entre los hombres que tienen relaciones con otros hombres, las tasas de infección se han mantenido estables (6). A la luz de estos hallazgos surgen algunas preguntas importantes relacionadas con la eficacia de la terapia ARMA, que se encuentra ampliamente disponible en Australia, entre ellas si ofrece cierto grado de protección contra la infección, al menos en el nivel poblacional.

Algunos datos preliminares, sin embargo, parecerían indicar que la terapia ARMA reduce la carga vírica en la sangre, pero que no tiene el mismo efecto en la concentración de partículas víricas presentes en el semen. Por consiguiente, sigue poniéndose en tela de juicio el valor de la terapia en la prevención secundaria de la infección (7).

Por otra parte, cierta información anecdótica indica de manera contundente que en otros lugares del mundo están ocurriendo cambios significativos. Durante una reunión del Programa Conjunto de las Naciones Unidas sobre el Sida (ONUSIDA) -llevada a cabo recientemente en Ginebra y referida al impacto de las terapias preventivas- los participantes de Uganda, Tailandia y Zambia informaron que en sus países el advenimiento de los nuevos tratamientos está cambiando la percepción que se tiene sobre la gravedad de la enfermedad (8). En Londres, Paul Ward del Terrence Higgins Trust describió algunas de las creencias y malentendidos que cada vez son más comunes: que el VIH es tratable y por ello no hay necesidad de protegerse durante las relaciones sexuales; que aun cuando los actuales tratamientos no son perfectos, pronto van a serlo; que actualmente se dispone de una pastilla para el día después del coito que protege contra la infección por VIH; que la mayoría de las personas infectadas por VIH están en tratamiento y por ello no son infectantes; y que si la carga vírica de la pareja no es detectable, se puede tener con ella contacto sexual sin protección (9). Aun más recientemente, algunos grupos de activistas expresaron su descontento porque durante las discusiones de la Asamblea Mundial de la Salud un ministro de salud centró su intervención básicamente en la necesidad de reforzar la prevención. Este enfoque fue calificado de "obsoleto" por parte de quienes sostienen que la atención de los pacientes centrada en tratamientos farmacológicos constituye la única solu- ción viable para enfrentar "la catástrofe del sida". No existen testimonios documentados acerca de cuán extendidas se encuentran las actitudes y creencias que subvaloran la prevención, ni tampoco está claro cómo puedan influir sobre el comportamiento, pero el hecho de que se estén verbalizando constituye un motivo de alarma. Todos estos hallazgos demuestran que es fundamental continuar subrayando la importancia de la prevención.

La prevención secundaria es esencial, incluso para las personas que ya están infectadas por el virus. Tal prevención consiste en intervenciones y programas dirigidos a ayudar a los individuos seropositivos a protegerse contra las infecciones oportunistas, y a proteger a otros contra la infección (10). Varios trabajos recientes señalan que para lograr reducciones sostenidas de la infección es necesario impulsar más vigorosamente la educación del público y la realización de pruebas detectoras, de manera que un mayor número de personas conozcan su estado serológico (11). También se ha señalado que los mensajes preventivos, los programas de educación pública y otras acciones afines deberían variar según vayan dirigidos a personas seropositiva o no (12). Sin embargo, a fin de que estas acciones den los resultados esperados, los trabajadores de la salud participantes deben poseer los conocimientos y destrezas que hacen falta, por un lado, para desempeñarse efectivamente en la evaluación de riesgos y provisión de asesoramiento y, por el otro, para cambiar la percepción de que el cuidado clínico y la prevención pertenecen a compartimentos separados en vez de ser puntos en un continuo.

\section{Consecuencias sociales de la terapia ARMA}

Hay adicionalmente otras preocupaciones de carácter más general relacionadas con la existencia de tratamientos nuevos. Por ejemplo, ¿conseguirán reducir los malos entendidos, los prejuicios y la discriminación hacia las personas infectadas por el VIH o, contrariamente, los aumentarán? Ya se dispone de pruebas de que conforme mejoran las condiciones de salud de las personas que reciben terapia ARMA, su deseo sexual o libido, hasta entonces drásticamente disminuido, comienza a normalizarse. ¿Cómo se interpretará el hecho de que una persona seropositiva al VIH aspire a reanudar una vida sexual activa, sin una educación pública que enseñe a los demás a comprender los cambios que están ocurriendo? Ante la ausencia de programas que promuevan percepciones positivas hacia quienes están infectados, no es realista suponer que la respuesta pública a la noticia de que las personas seropositivas están volviéndose más activas sexualmente vaya a ser favorable y solidaria. 
De hecho, en el marco de las actitudes sexofóbicas y homofóbicas prevalecientes en muchas sociedades, se da ya una marcada distinción en el trato a las personas que se infectaron a través de sus prácticas sexuales y las llamadas "víctimas inocentes" (receptores de transfusiones; recién nacidos infectados in utero; mujeres infectadas por su compañero, etc.). Esta demarcación implica que se reconoce a un grupo como responsable, culpable de su propia infección, adquirida a través de prácticas "vergonzosas" o "condenables". No es raro que en los debates sobre la disponibilidad de fármacos para la terapia ARMA en localidades con recursos limitados, las prioridades se inclinen hacia las "víctimas inocentes". En este sentido los dilemas éticos, más que ser analizados a la luz de los principios de equidad, utilidad o mérito relativo, se dilucidan sobre la base del prejuicio, la intolerancia y la ignorancia.

La respuesta de la comunidad ante la información relacionada con la terapia ARMA básicamente ha consistido en presionar para facilitar el acceso a los fármacos antirretrovíricos, con poco énfasis o ninguno en otros elementos que constituyen el cimiento y los pilares del manejo clínico de la infección y del control de la epidemia. El reconocimiento de los resultados positivos conseguidos con la terapia ARMA, aunado a los modelos sociales prevalecientes sobre la atención de la salud (paradigma curativo-reparador), ha reforzado la creencia de que el manejo de la infección por VIH tiene que centrarse en la terapia farmacológica. Ciertas medidas, que además de tener un efecto terapéutico agregan un componente preventivo, como los hábitos higiénicos y dietéticos y la profilaxis de infecciones oportunistas, han sido totalmente descuidadas, aun en localidades con recursos muy limitados, donde la reivindicación se ha tornado monotemática: acceso a fármacos antiretrovíricos. La presión sobre el sector de la salud para que garantice el acceso a la terapia antirretrovírica llega a constituir una carga, a menudo insostenible, que exige una reducción drástica de las escasas partidas destinadas a acciones de prevención (primaria y secundaria).

Una respuesta comunitaria que se observa en contextos con recursos muy limitados está basada en la solidaridad y la buena voluntad hacia el "reciclaje" de fármacos antirretrovíricos. Existen redes bien establecidas y muy activas que facilitan la donación y distribución de medicamentos, particularmente desde países desarrollados hacia los más necesitados. Desafortunadamente, el funcionamiento de este tipo de redes se ve obstaculizado por diversos factores: falta de regularidad de las donaciones (habitualmente fármacos sobrantes o descartados); restricciones aduaneras; ausencia de infraestructura en la localidad receptora para realizar el diseño y monitoreo de la terapia y, finalmente, la continua incertidumbre acerca de la sostenibilidad de la respuesta. Así, una respuesta muy bien intencionada puede involucrar graves problemas relacionados con las dificultades prácticas para tomar decisiones clínicas, mantener el esquema de tratamiento durante tanto tiempo como sea necesario, prevenir el desarrollo de resistencia a alguno o varios de los fármacos administrados y, no menos importante, realizar un control escrupuloso de la calidad y las formas de distribución de los productos donados. La adecuada orientación de estas respuestas, que pueden tener efectos indeseables no previstos, exige de un gran esfuerzo educativo entre los miembros de la comunidad, a fin de facilitar la comprensión de los pros y los contras de instaurar esquemas de tratamiento a largo plazo basados en donaciones.

La experiencia ha demostrado que el trabajo de educar a la comunidad no puede limitarse a la transmisión de información factual sobre VIH o sobre los recursos disponibles para hacer frente a la epidemia. Es necesario poner en marcha acciones destinadas a cambiar los modos en que se percibe el cuidado de la salud, esto es, incidir sobre la "cultura de la salud" de las personas.

\section{La prevención después de la exposición}

Los mensajes educativos siempre han subrayado la importancia de la prevención primaria por medio de relaciones sexuales protegidas, así como del uso seguro de drogas intravenosas y del apoyo y cuidado de las personas con la infección por VIH o sida. Recientemente se ha incorporado al discurso y a la agenda preventiva la llamada "prevención después de la exposición" (pde) o medicación profiláctica (13). En tanto que la zidovudina usada de forma profiláctica ha mostrado ser eficaz para disminuir el riesgo de la infección por VIH en trabajadores de la salud después de accidentes con agujas contaminadas, no sabemos si existe un efecto protector similar en el caso de las relaciones sexuales (14). A pesar de ello, la medicación profiláctica, con una combinación de zidovudina, lamivudina y un inhibidor de la proteasa, está siendo empleada por algunos médicos en personas que han tenido contacto sexual sin protección con una persona infectada (15).

Cuando se trata de decidir si la medicación profiláctica es una fuente potencial de protección, se hace necesario considerar diversos factores, entre ellos los costos (16). Por otra parte, la eficacia de este tipo de medidas puede reducirse considerablemente cuando se usan en exceso después de la exposición. Otros aspectos que es preciso tomar en cuenta para decidir si la medicación profiláctica se justifica o no son el estadio de la infección en la persona seropositiva, su carga vírica, la presencia de lesiones durante 
el coito, alguna infección de transmisión sexual concurrente y la presencia de sangre menstrual durante el coito cuando la pareja es mujer.

La medicación profiláctica plantea una serie de preguntas para las cuales de momento no existen respuestas. Es posible que su uso generalizado fomente la creencia de que existe un tratamiento rápido contra la infección por VIH o el sida; que lleve a un aumento del sexo sin protección si el tratamiento se vuelve ampliamente accesible, y que conduzca a quienes tengan actividades sexuales riesgosas para la transmisión del VIH a usar medicamentos antirretrovíricos antes de tener relaciones sexuales, si creen que aquellos pueden tener un efecto protector (17).

Es poco probable que la medicación profiláctica sea eficaz en todos los casos. ¿Cómo balancear, entonces, el riesgo del éxito en el tratamiento contra el riesgo de infectarse? ¿Y qué hay del impacto físico y psicológico de recibir medidas de prevención después de la infección? Estas, a la par del reto que supone garantizar que la medicación profiláctica sea accesible equitativamente, con independencia de la edad, orientación sexual y forma en que se practicó el sexo sin protección, son algunas de las muchas preguntas que siguen sin respuesta. Es preciso que las voces de los grupos y comunidades más vulnerables sean escuchadas en estos debates y lograr que las respuestas a las preguntas formuladas no se den en privado, sino con la participación de los usuarios de los servicios de salud y los grupos afectados (18).

\section{Vale más prevenir que curar}

Cada vez que muere una persona como consecuencia de las complicaciones relacionadas con el sida, uno lamenta que se haya perdido una vida humana por falta de los recursos terapéuticos necesarios que podrían haberle salvado la vida. Si se considera que el costo de cualquier vida es incalculable, resulta a veces incomprensible que no se inviertan los recursos financieros necesarios para salvar o al menos prolongar la vida. Un argumento frecuentemente empleado es que dichos recursos no se encuentran disponibles y, en el caso de la terapia antirretrovírica muy activa, que el costo de los medicamentos y del control (monitoreo) de los mismos está más allá del alcance de los sistemas de salud locales afectados. Este argumento, para ser válido, debería acompañarse de acciones consecuentes, como el desplazamiento del énfasis en la provisión de servicios curativos y clínicos por profesionales cada vez más especializados hacia un mayor número de acciones preventivas y de promoción de la salud, así como hacia eliminar barreras políticas y culturales que dificultan la "minimización de daños". A los profesionales de la salud les cabe un papel primario en la reorganización de los servicios por ser figuras públicas que gozan de la credibilidad para ayudar a transformar atavismos seculares en materia de atención de la salud, malentendida esta solo como cura de las enfermedades (21).

El acento en la prevención - que exige compromisos en todos los niveles del sistema de salud- permite reducir los costos asociados con la enfermedad y la invalidez, no solamente financieros, sino también sociales, familiares y emocionales. Muchos programas privados de atención de la salud han ampliado la cobertura de los servicios preventivos porque se ha demostrado que el costobeneficio de estos servicios es mayor en relación con el enfoque reparador, sin contar el sufrimiento innecesario que puede ser evitado. La mejor manera de enfrentar el desafío que representa la epidemia de infección por VIH y sida sigue siendo prevenir la aparición de nuevas infecciones.

Para quienes ya se encuentran infectados podría parecer frívolo y hasta irónico hablar de prevención. En realidad, como se mencionó anteriormente, aun en los casos de las personas seropositivas la prevención tiene una importancia crucial para protegerlas de las infecciones oportunistas y contener la diseminación de la infección. Sin duda alguna, la prevención de la infección por VIH y del sida sigue siendo crucial, incluso si se dispone de terapia antirretrovírica.

Agradecimiento. Los autores están muy agradecidos con Blanca Rico por su ayuda con la traducción. Ciertos elementos de esta ponencia han aparecido en Actualizaciones en sida (Argentina), Volumen 6, No. 22, pp. 228-231, y en ETS-Sida (México), Volumen 4, pp. 85-88. Están reproducidos aquí con el permiso de los editores.

\section{SYNOPSIS} Antiretroviral agents against HIV and AIDS are
now available: Is prevention still necessary?

New antiretroviral agents have radically changed the treatment of HIV infection and AIDS. In many patients, this treatment has produced substantial improvement, as well as marked reductions in circulating viral particles. Furthermore, there has been a decrease in mortality from AIDS complications resulting from the use of very active retroviral therapy (VART). A source of concern, however, is the current perception that the disease, though not yet curable, can be successfully treated with this new therapy, since this notion could be changing the public's attitude toward the continued need for preventive measures. 


\section{REFERENCIAS}

1. United Nations Program on Aids. Relationships of HIV declines in Thailand to behavioural change. Geneva: World Health Organization; 1998. (Best Practice Collection).

2. United Nations Program on Aids. Expanding the global response to HIV/ AIDS through focussed action. Geneva: World Health Organization; 1998.

3. Van deVen P, Crawford J, French J, et al. Increase in UA/C among Sydney gay men in 1996-1998. Póster presentado en la 1998 XII World AIDS Conference, Ginebra, Suiza.

4. Dilley J, Woods W, McFarland W. Are advances in treatment changing views about high-risk sex? [Carta]. New Engl J Med 1997;337(7):501-502.

5. Australian Federation of AIDS Organisations. Briefing on reported increase in incidence of unprotected anal sex with casual partners in gay men sampled in Sydney. Sydney: AFAO; 1997.

6. Temoshok L, Frerichs R. Secondary HIV prevention. Focus: a guide to AIDS research and counselling 1998;13(7):1-4.

7. Vernazza PL, Gilliam BL, Dyer J, Fiscus SA, Eron JJ, Frank A, et al. Quantification of HIV in semen: correlation with antiviral treatment and immune status. AIDS 1997;11(8):987-993.
8. United Nations Program on Aids. Report from a meeting. New Challenges for HIV Prevention. Geneva: UNAIDS; 1998.

9. Jones J. The impact of new treatments on HIV prevention: conference report. En: Myths and misunderstanding. London: Health First, Terrence Higgins Trust, Camden and Islington Community Health Service, NHS Trust/National AIDS Manual; 1998

10. National Centre in HIV Epidemiology and Clinical Research. Australian HIV Surveillance Report (Sydney)1998;14:2

11. Cates W, Chesney M, Cohen M. Primary HIV infection: a public health opportunity. Am J Public Health 1997;87: 1928-1930.

12. Senterfitt W. Positive images: primary prevention for people with HIV. Focus: a guide to AIDS research and counselling 1998;13(7):5-6.

13. Katz M, Volberding J. Postexposure treatment of people exposed to the human immunodeficiency virus through sexual contact or injecting drug use. New Engl J Med 1997;336:1097-1100.

14. Daily J. Postexposure prophylaxis for HIV. AIDS Clin Care 1997;9(8):13.

15. Katz M, Geberding J, Boswell S. Postexposure prophylaxis: a roundtable discussion. AIDS Clin Care 1998;10(2):1-3.
16. Wolfson J. The dangerous financial politics of AIDS "cocktail" therapies. J Health Care Finance 1997;24(1):59-63.

17. Australian Federation of AIDS Organisations. The implications of the availability of HIV exposure prophylaxis. Advice sheet for members. Sydney: AFAO; 1998.

18. Nicholson N, Fysh G. Ethical and practical problems of a "morning after" pill for HIV. National AIDS Bul 1999;11(5): 26-27.

19. Pomerantz R. Residual HIV-1 disease in the era of highly active antiretroviral therapy. New Engl J Med 1999;340(21): 1672-1674.

20. Furtado MR, Callaway DS, Phair JP, Juntsman KJ, Stanton JL, Macken CA, et al. Persistence of HIV-1 transcription in peripheral-blood mononuclear cells in patients receiving potent antiretroviral therapy. New Engl J Med 1999;340(21): 1614-1622.

21. Nutbeam D, Blakey V. El concepto de promoción de la salud y la prevención del sida: una base integrada y general para la acción en los años noventa. En: Promoción de la salud: una antología. Washington, D.C.: Organización Panamericana de la Salud; 1996. (Publicación científica 557). 